\title{
LA INTEGRACIÓN DE JOSÉ DONOSO A LA PLATAFORMA DEL BOOM: INTERCAMBIO EPISTOLAR INÉDITO DE JOSÉ DONOSO CON EMIR RODRÍGUEZ MONEGAL Y CARLOS FUENTES EN LA DÉCADA DEL 60
}

\author{
POR \\ María Laura Bocaz Leiva \\ University of Mary Washington ${ }^{1}$
}

\begin{abstract}
A más de cincuenta años de la década que asociamos con el Boom de la novela latinoamericana, Historia personal del Boom (1972) se ha convertido en uno de los textos fundamentales para nuestro acercamiento crítico a este período, junto con "El 'Boom'en perspectiva” (1979) de Ángel Rama, y “El 'boom'de la novela latinoamericana” (1972) de Emir Rodríguez Monegal. A diferencia de los textos de ambos críticos, la Historia de Donoso se escribe y presenta abiertamente como un recuento que surge del recuerdo y de la propia experiencia de un escritor que si bien desconoce cuál es el lugar que ocupa dentro del Boom, se considera capacitado para dar su testimonio de ese período:
\end{abstract}

\begin{abstract}
Me cuento entre aquellos que no conocen los deslindes fluctuantes del boom y me siento incapaz de fijar su hipotética forma ... y para qué decir desentrañar su contenido. Pero sea cual fuere la posición y categoría de mi obra dentro de la novela hispanoamericana contemporánea, mis libros han aparecido en y alrededor de la década del sesenta, y así me siento ligado a, y definido por, las corrientes y mareas del ambiente literario de nuestro mundo, cambios determinados por la publicación de ciertas novelas que incidieron poderosamente en la visión y en el quehacer de este escriba. Dar mi testimonio personal de esas obras, decir cómo las sentí y cómo las sigo sintiendo, contar de qué manera vi sobrevenir los cambios desde el ángulo que a mí me tocó, y qué carácter tuvieron para mí esos cambios ... será, más que nada, el propósito de estas notas $(18){ }^{2}$
\end{abstract}

1 Agradezco la guía y apoyo de Daniel Balderston y Brian Gollnick durante mi trabajo e investigación con los José Donoso Papers.

2 Quiero subrayar que una revisión de las reseñas sobre Historia personal del Boom publicadas en los setenta deja en evidencia dos actitudes por parte de los críticos frente a las peculiares características del texto. Por una parte quienes discuten su naturaleza, comentando su componente histórico y anecdótico (Hasset), y quienes por el contrario, se limitan a ofrecer una síntesis parcial de los postulados de Donoso (Gerdes, McMurray). John Hassett en 1973 subraya la naturaleza conflictiva de Historia personal definiéndolo como un comentario que trata de evaluar a la vez que reconstruir desde la propia experiencia, el fenómeno en cuestión: "Donoso's Historia personal is a very uneven book which must be approached from two different perspectives. One is that of a commentator who is attempting to evaluate a particular 
No sólo a partir del título, sino que insistentemente a lo largo de su recuento, Donoso se encarga de subrayar la subjetividad de su testimonio. Por ejemplo, en la siguiente advertencia al lector, autodefensa ante posibles cuestionamientos, Donoso define el objetivo de su texto explicitando sus limitaciones: "Debo dejar en claro que no es la intención de estas notas definir el Boom. Nada de lo que digo aquí pretende tener la validez universal de una teoría explicativa que asiente dogmas: ... hablo aqu aproximadamente, tentativamente, subjetivamente, ya que prefiero que mi testimonio tenga más autenticidad que rigor" (17-18). ${ }^{3}$

literary phenomenon; the other, that of a creative writer who strives to re-create for the reader the cultural literary phenomenon; facasa: "As a scholarly contribution to our knowledge of he "boun as literary autobiography the work does indeed have merit. We learn far more about José Donoso than we do about the "boom" and it is this aspect of the book that saves it" (110). Para Hasset los motivo de su fracaso en tanto historia radican en que el escritor no logra superar la anécdota personal: "Donoso lacks the necessary critical insight and objectivity. Perhaps by borrowing the term personal from Chile's renowned critic Alone $[\ldots]$ he hopes to justify the highly subjective way in which he treats his subject, bu too often his comments never rise above the level of mere gossip and name-calling" (108). Por ejemplo, le critica el desplazar un análisis de las diferencias entre las tres novelas que Donoso propone como hito dentro del desarrollo de la nueva novela (Ravuela, La ciudad y los perros, Cien años de soledad) con nécdotas centradas en su relación con los escritores: "The validity of designating these three novels

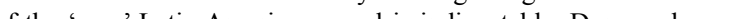
eglects to take us one ste finthe the new' Latin A chosen represents a distinct stage. Instead of hidicating specifically what changes have occurred from on novel to the other, the author simply supplements his initial statements with anecdotes about the writer in question. To the frustration of the reader Donoso continues in this vein throughout the entire book" (109-110).

También en 1973, George McMurray califica Historia personal como un testimonio tanto de la actividad profesional de Donoso en las décadas del cincuenta y sesenta, como de sus impresiones del Boom. A diferencia de Hassett, no discute la naturaleza del texto ni cuestiona su acercamiento, sino que subraya su aporte a nuestro entendimiento del Boom así como de la obra y figura de Donoso, mediante una síntesis comentada de una selección de hechos discutidos por el escritor en su Historia personal. A saber, 1 aisla e

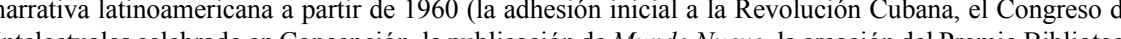
Intelectuales cecleber Breve, la agencia de Carlos Fuentes). Por último, el clímax del Boom y las causas de la desunión entre los escritores.

Dick Gerdes (1977) por su parte, también opta por resumir algunos de los planteamientos de Donoso: unidad inicial de los escritores, el hecho de que la existencia del Boom se deba principalmente a sus detractores, los nombres y fechas que a juicio de Donoso fueron importantes para el nacimiento del Boom. No obstante, a diferencia de McMurray, subraya en su reseña lo que considera una falencia del texto: el no incorporar en su recuento el aporte de Asturias, Carpentier, Yáñez, Sábato, Marechal y Rojas a la internacionalización de la novela regional entre 1946 y 1951 (445).

Concuerdo con Verónica Cortínez en la escasez de estudios monográficos dedicados a Historia personal (16). A mi parecer Jacques Joset es quien ha ofrecido el acercamiento crítico más completo de este texto a través de "El imposible Boom de José Donoso" (1982) y "La estrategia autobiográfica de José

Revista Iberoamericana, Vol. LXXIX, Núms. 244-245, Julio-Diciembre 2013, 1049-1068 ISSN 0034-9631 (Impreso) ISSN 2154-4794 (Electrónico)
A diferencia de "las notas" abiertamente subjetivas de Donoso, donde el autor no discute ni resuelve su posición dentro del Boom por considerarla una tarea que le corresponde a los especialistas, los textos de Rama y Emir Rodríguez Monegal ofrecen al lector una revisión crítica de este período en la que Donoso figura en tanto escritor. A partir del libro de Rodríguez Monegal se puede inferir que Donoso es un protagonista del Boom básicamente por haber recibido dos importantes premios internacionales relacionados con el Boom de la novela latinoamericana (el de la Fundación William Faulkner por Coronación (30-31) y el de Seix Barral por su manuscrito del Obsceno pájaro (23) ) por la publicación de textos inéditos en Mundo Nuevo y por el hecho de que el Obsceno pájaro constituyera una de las tantas novelas donde se hace evidente el impacto de Rayuela (84-85).

El texto de Rama, a diferencia del de Rodríguez Monegal, no resuelve para el lector la posición que Donoso-escritor ocupa en el Boom. Por el contrario la problematiza al apuntar "la pluralidad de las listas" disponibles (24):

Donoso en Historia personal del 'boom”' (1986). En el primero, Joset propone entender este texto como una autobiografía literaria, pero donde "por más personal que sea, se intenta definir el estatuto histórico del boom y sus componentes" (92). De este modo, subraya por una parte, que a pesar de que Donoso declare no querer definir el boom, relegando la tarea a los especialistas, el texto presenta reiterativas firmaciones sobre su "existencia y unida" (92). Por otra, que las contradicciones que presenta result al movimiento literario] obedece en buena parte a las leyes de cuyo auto-protagonista nunca presume de ser 'dueño de un sistema monolitico que pretende explica los fenómenos literarios' (96)" (101). Por último, Joset propone Historia personal como una referencia insustituible para el estudio del Boom (101) al subrayar que a pesar de su carácter subjetivo y de las contradicciones que presenta (96) constituye una historia, ya que ofrece una descripción de una serie de "fenómenos literarios, distribuidos a lo largo de un eje cronológico" (95) que nos ayudan a "reconstruir el trazado de algunas líneas" del Boom (93). Posteriormente en "La estrategia autobiográfica" (1986) Joset amplía su clasificación original de Historia personal como autobiografía literaria al añadirle el calificativo de profesional (642). Subraya la doble intencionalidad del libro -defender una tesis y ofrecer una autobiogréfica literaria- así como la estrategia utilizada por el escritor para otorgar credibilidad a su

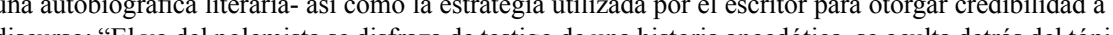
de la humildad para acreditar la tesis" (648). En el cuerpo de su estudio, Joset identifica y analiza los diferentes elementos autorreferenciales del texto (642-645), subraya la relación dialéctica entre el yo y el nosotros -a saber, los escritores que comparten con Donoso las ideas relacionadas con "la literatura de continente" (645)- resaltando que se observa una confusión de "la aventura individual con la colectiva" (645). Identifica y analiza los capítulos 4 y 5 donde Donoso relega al yo a un segundo plano del discurso, para "contestar al argumento de los adversarios del boom" (646), resaltando a su vez, la hibridez del quinto capítulo por contener "el catálogo comentado de las lecturas que contribuyeron a la formación novelística del escritor: Rayuela, Sobre héroes y tumbas, La ciudad y los perros" (646). Asimismo, resalta la peculiaridad del tercer capítulo de centrarse en Carlos Fuentes, los efectos que la lectura de La región mas transparente tuvieron en el escritor (647) asi como el rol del escritor mexicano tuvo en su formación como escritor (647).

Para un acercamiento a Historia personal centrado en la utilización de la forma del ensayo, ver Verónica Cortínez "La parroquia y el universo: Historia personal del Boom de José Donoso".

Revista Iberoamericana, Vol. LXXIX, Núms. 244-245, Julio-Diciembre 2013, 1049-1068 ISSN 0034-9631 (Impreso) ISSN 2154-4794 (Electrónico) 
Teniendo en cuenta estos textos [aquellos donde aparecen las enumeraciones de Carlos Barral, Vargas Llosa, Cortázar, Fuentes y el propio Donoso en su Historia personal (25-26)] puede hacerse más comprensible que yo haya satirizado al boom definiéndolo como el club más exclusivista que haya conocido la historia cultural de América Latina, un club que tiende a aferrarse al principio intangible de sólo cinco sillones y ni uno más, para salvaguardar su vocación elitista. De ellos, cuatro son, como en las Academias 'en propiedad’: los correspondientes a Julio Cortázar, Carlos Fuentes, Mario Varas Llosa y Gabriel García Márquez. El quinto queda libre para su otorgamiento: lo han recibido desde Carpentier a Donoso, desde Lezama Lima a Guimarães Rosa. (26)

Mediante el presente artículo me he propuesto entablar un diálogo entre la posición en la que Donoso ha quedado dentro del Boom a partir de los tres textos que desde la década del 70 han marcado nuestro entendimiento de este período, y la correspondencia personal de José Donoso con Emir Rodríguez Monegal y Carlos Fuentes, con el objetivo de subrayar el papel que ambos jugaron en la entrada de Donoso al escenario del Boom durante la década del 60. Mi punto de partida es que la incorporación crítica de la correspondencia personal del escritor permite cuestionar el fenómeno de la consagración de Donoso en términos cronológicos, al revelar a un José Donoso que no se suma al Boom a fines de la década del sesenta-principios de los setenta, sino que es tempranamente incorporado por dos figuras cruciales, Emir Rodríguez Monegal y Carlos Fuentes. La correspondencia entre Donoso y Fuentes analizada pertenece a los José Donoso Papers de las universidades de Iowa y Princeton, en tanto las cartas entre Donoso y Rodríguez Monegal, a los José Donoso Papers y Emir Rodríguez Monegal Papers de la colección de Princeton. En el caso de Rodríguez Monegal, también incorporo a la discusión la presencia de Donoso en Mundo Nuevo durante el período de su dirección (1966-1968). ${ }^{4}$

Entre las diferentes vivencias que Donoso incorpora en ese testimonio que no pretende definir las coordenadas del Boom, responder preguntas ni resolver polémicas, resulta particularmente interesante su acercamiento a Emir Rodríguez Monegal y Mundo Nuevo, ya que en esa Historia que ha calificado de personal, el "hablar subjetivamente" sobre el crítico uruguayo y la revista que dirigió hasta 1968, se restringe a subrayar su agencia en la circulación de la literatura latinoamericana y en suscitar la cohesión de un grupo restringido de escritores, entre los que Donoso no se incluye explícitamente como uno de los favorecidos:

[...] ejerció, durante los años en que con talento y discriminación la manejó [...] un papel decisivo en definir una generación. [...] Mundo Nuevo fue la voz de la literatura latinoamericana de su tiempo, y para bien o para mal, y con todo el riesgo que implica,

\footnotetext{
4 No he incluido en este trabajo la correspondencia de Donoso contenida en los Carlos Fuentes Papers, ya
que esta caja se encuentra cerrada al investigador hasta enero del año 2021 o bien dos años después del
} allecimiento del escritor.

Revista Iberoamericana, Vol. LXXIX, Núms. 244-245, Julio-Diciembre 2013, 1049-1068 ISSN 0034-9631 (Impreso) ISSN 2154-4794 (Electrónico) estoy convencido que la historia del 'boom' en el momento en que presentó su aspecto más compacto, está escrita en las páginas de Mundo Nuevo, hasta el momento en que Emir Rodríguez Monegal abandonó su dirección. (121-22)

El riesgo que Donoso teme correr mediante sus declaraciones radica justamente en ese mostrarse a favor de la labor de Rodríguez Monegal y de la función de Mundo Nuevo en la consolidación del Boom. En optar por dejar a un lado las acusaciones que desde 1965 la revista y su director recibieron, para subrayar el rol que a su juicio ambos tuvieron en la formación y fortalecimiento del Boom. No obstante lo anterior, si se complementa esa declaración que el escritor califica de osada, con el testimonio que ofrece su correspondencia con Rodríguez Monegal, ésta sorprende por su brevedad y tono impersonal al eclipsar por completo dos hechos que el intercambio epistolar deja instantáneamente en evidencia. A saber, el interés temprano de Rodríguez Monegal en la obra y figura de Donoso, así como el apoyo constante e incondicional que le brinda ${ }^{5}$

Donoso también incluye en su testimonio, la función que a su juicio tiene Carlos Fuentes en la internacionalización de la literatura latinoamericana, presentándolo como un indiscutido impulsor del Boom: "Mirando, como siempre, el fenómeno desde mi punto de vista personal, aparece Carlos Fuentes como el primer agente activo y consciente de la internacionalización de la novela hispanoamericana de la década del sesenta" (49). Como se puede ver a continuación, Donoso se preocupa de destacar que esa agencia que le atribuye no se limita a las repercusiones que entonces tuvo la obra del escritor mexicano en sus contemporáneos: "no sólo por el estímulo literario de sus primeras novelas, sino también por su generosidad [...] Carlos Fuentes ha sido uno de los factores precipitantes del boom" (64). Esa generosidad que Donoso exalta, se define un par de páginas más adelante cuando lo señala como el mayor responsable del Boom entre los escritores: "Carlos Fuentes es sin duda el que más se mueve, el que más congresos organiza, el que más cartas recibe, y el que más proyectos inventa, proyectos destinados a irradiar su luz sobre gran parte de los escritores de su generación" (Anagrama: 65). ${ }^{6}$

En oposición a las sucintas declaraciones de Donoso en relación a Rodríguez Monegal, cuando se refiere a Fuentes su testimonio no se limita a subrayar la agencia del escritor mexicano en la maquinaria del Boom, sino que destaca la influencia directa

5 Para una exhaustiva revisión del conflicto que genera la posibilidad de que la revista fuera financiada en parte por la CIA, ver María Eugenia Mudrovic, Mundo Nuevo: cultura y guerra fría en la década del 60 9-94.

6 Cito la primera edición de Historia personal (Anagrama 1972), ya que esta cita no aparece -al menos contrastiva entre las ediocios de Augiero comenza por las útinas dos, ya que a jazgar por la contapa de la primea edición en Alfaguara, Donoso puede haber intervenido el texto: "La edición de 1987 incorporó los apéndices del autor y de María Pilar Donoso" N.pag.

Revista Iberoamericana, Vol. LXXIX, Núms. 244-245, Julio-Diciembre 2013, 1049-1068 ISSN 0034-9631 (Impreso) ISSN 2154-4794 (Electrónico) 
que éste tuvo en su carrera así como en la circulación de su propia obra: "Fue Carlos Fuentes, el que sabiéndome asfixiado de mi país, propuso mi nombre a Bob Wool para que me invitara al Symposium de Chichen-Itzá.” (108). Así mismo, se refiere a la gestión de Fuentes para que Coronación fuera traducida al inglés: "recibí una generosa carta de Carlos Fuentes en que manifestó su entusiasmo por mi novela: 'Encuentro absurdo que esta novela no se conozca más y que no se haya traducido. Mándasela a mi agente literario en Nueva York, Carl D. Brandt, y yo le escribiré para ver qué puede hacer por ella"' $(63){ }^{7}$

La brevedad y el tono impersonal con el que Donoso califica la labor de Rodríguez Monegal y la revista que dirigía, se puede atribuir a que su Historia personal fue escrita y publicada cuando aún estaba presente la polémica que se generó ante la posibilidad de que parte de los fondos de la revista fueran proveídos por la CIA. Una carta enviada por Donoso al crítico uruguayo del 11 de octubre de 1972, sugiere que por lealtad a Rodríguez Monegal optó por hablar sucintamente de él y la revista: "No temas por lo de MUNDO NUEVO en mi libro HISTORIA PERSONAL DEL BOOM [...] Lo único que en ese libro afirmo es que MUNDO NUEVO fué (sic), durante el mandato de Emir, el órgano que cohesionó y le dio forma al Boom en su momento más rico y creativo. I do no scandalmongering of any kind, y te dejo como un rey" (11/10/72 Emir Rodríguez Monegal Papers, PU). De este modo, interpreto la reducción que Donoso hace de la figura de Rodríguez Monegal a su rol de director de Mundo Nuevo, como la estrategia que le permitió incluirlo como una de las figuras esenciales del Boom, sin correr el riesgo de perjudicarse.

Otro aspecto que la correspondencia sugiere es que el apoyo que Donoso recibe se sustenta en la posición privilegiada que como docente, crítico y director de Mundo Nuevo, Rodríguez Monegal ocupa en el contexto de la producción y circulación de la literatura latinoamericana. El intercambio epistolar se inaugura con dos cartas de 1965 , archivadas en la Universidad de Iowa. Para entonces, Emir Rodríguez Monegal forma parte del departamento de Romance Languages and Literatures, en una universidad norteamericana, y Mundo Nuevo es un proyecto a punto de concretarse: "Por Claudio Campuzano obtengo tu dirección y te digo: El proyecto de la revista en París es una realidad" (7/11/65 José Donoso Papers UI). Donoso, por su parte, está próximo a dirigirse a Iowa City para participar en el Writers' Workshop de la universidad de dicha ciudad.

La utilización de las comillas en el fragmento citado, permite inferir que Donoso ha reproducido literalmente la carta enviada por Fuentes. No obstante, como demostraré más adelante, al contrastar este fragmento con la epístola original se observan variantes. Lo más probable es que Donoso durante e proceso de composición de su Historia, reprodujera la carta recurriendo a la memoria y no a partir de la original, puesto que ésta ya formaba parte del archivo de la Universidad de Iowa.

8 Donoso estuvo en la Universidad de Iowa en tres ocasiones. Primero, cuando participó en el Writers' Workshop entre 1966 y 1967 y luego en el International Writing Program en 1968 y 1991.

172 Revista Iberoamericana, Vol. LXXIX, Núms. 244-245, Julio-Diciembre 2013, 1049-1068 ISSN 0034-9631 (Impreso) ISSN 2154-4794 (Electrónico)
Ambas cartas dejan ver cómo Rodríguez Monegal aprovecha su posición como miembro de un departamento de literatura de una universidad prestigiosa, para difundir e introducir a Donoso y su obra. En la primera, comenta entusiasmado el interés que sus cuentos han generado en uno de sus alumnos: "tengo un alumno escribiendo un paper sobre tus narraciones. Ya oirás de él" (18/3/65 José Donoso Papers UI). En la segunda, le informa de la posibilidad de ser invitado a la universidad: "Ya propuse a la gente del Departamento de Romance Languages and Literatures que te invite a venir aquí, a dar una conferencia.... Todos están encantados con la idea de que vengas y quieren saber cuándo sería posible" (31/3/65 José Donoso Papers UI). ${ }^{9}$

Destaco las estrategias de inserción de la obra de Donoso en un programa académico, porque refuerza lo que una revisión de los números de Mundo Nuevo, publicados bajo la dirección de Rodríguez Monegal, deja en evidencia. Esto es, que para el crítico, Donoso constituye una figura clave de esa generación de nuevos escritores que mediante Mundo Nuevo, y más tarde a través de otras revistas, decide difundir. Cabe señalar que la iniciativa de incorporar la obra de Donoso en el área académica continúa una vez que Rodríguez Monegal retoma su labor docente. Ejemplo de ello se encuentra en una carta escrita tres años después, en la que comenta a Donoso su inclusión en un curso que imparte:

No sabés lo caro que me estás costando. Te lo cuento: 1) Estoy dando un curso aquí sobre las relaciones entre la novela norteamericana y la latinoamericana y el primer autor que estudio eres tú ... en reacción con el viejito James y sus Aspern Papers=Spoils of Poynton, con algunas excursiones adicionales hacia La dama de espadas, de Puschkin y, es claro, Great Expectations. Los muchachos encantados con el paseo (11/10/68 José Donoso Papers PU).

En cuanto Mundo Nuevo comienza a funcionar, el apoyo que Donoso recibe por parte de Rodríguez Monegal adquiere una nueva dimensión. Lo que al director de la revista le interesa, a partir de entonces, es la colaboración de Donoso mediante su obra, experiencia como lector y crítico: “HHay alguna posibilidad de tener un cuento tuyo o algún pasaje excitante de tu novela para el primer número de la Revista? Saldría en marzo o abril. ... también te escribiré apenas tenga organizada la oficina en París porque estoy pensando en tí (sic) en terminos (sic) de una colaboración más estable y sobre todo de reseñas críticas, breves y con punch" (7/11/65 José Donoso Papers UI).

Esta petición se reitera dos meses más tarde: “creo que tu incorporación a la revista sería fundamental no sólo por el prestigio de tu nombre sino por la simpatía personal que nos une"(18/01/66 José Donoso Papers UI) y persiste incluso después de que Rodríguez

\footnotetext{
El crítico está haciendo referencia a la Universidad de Harvard donde fue Visiting Professor durante e semestre de Primavera de 1965. Ver carta de José Donoso del 18 de marzo de 1966 en los José Donoso Papers de la Universidad de Iowa.

Revista Iberoamericana, Vol. LXXIX, Núms. 244-245, Julio-Diciembre 2013, 1049-1068 ISSN 0034-9631 (Impreso) ISSN 2154-4794 (Electrónico)
} 
Monegal renuncia a la dirección de Mundo Nuevo. Una carta de agosto de 1968 deja constancia de que para el crítico su salida de Mundo Nuevo no es un impedimento para que Donoso publique fragmentos de la obra que entonces lo mantenía ocupado: "Me interesó mucho lo que me dices del famoso Obsceno Pájaro. ¿Por qué no me mandas un capítulo, así lo hago publicar aquí en la revista Imagen que es una de las mejores de América Latina? ... Tú sabes que tengo una predilección muy especial por tu obra y me gustaría seguir colaborando en su difusión. Así que decídete" (23/8/68 José Donoso Papers PU). ${ }^{10}$

Como señalé anteriormente, la colaboración que Rodríguez Monegal espera de Donoso para la revista que dirige, no se limita al envío de material inédito. Una carta que el crítico escribe a Donoso en junio del mismo año, deja constancia de su confianza en la lectura y conocimiento de Donoso, al invitarlo a escribir "algo sobre los jóvenes narradores mexicanos como [Salvador] Elizondo, Saiz [Gustavo Sainz], Inés Arredondo, Ulises Carrión". Junto con invitarlo, le aconseja en qué términos concretarla: “escrita con cierta severidad pero no hasta el punto de impedirte para siempre la entrada en México. ¿Qué te parece? Casi todos esos libros han sido publicados por Mortiz o por Era de modo que no te será difícil obtenerlos" (6/7/66 José Donoso Papers UI).

Una revisión de los números de Mundo Nuevo publicados hasta la renuncia de Rodríguez Monegal a la dirección de la revista, sin embargo, permite constatar que la participación de Donoso se limita al envío de una serie de avances de las obras en creación. Este hecho me invita a adjudicar la presencia de la obra de Donoso en Mundo Nuevo, principalmente a la iniciativa de Rodríguez Monegal, consecuencia que, como se verá a continuación, es el resultado de una serie de maniobras que el crítico lidera y a la que denomina la "operación Donoso".

La presencia de la obra de Donoso en Mundo Nuevo es particularmente sobresaliente en los números 12 (junio), 14 (agosto) y 15 (septiembre) de 1967. Una carta que Rodríguez Monegal envía a Donoso, el 13 de abril de 1967, permite acceder al preámbulo de esas sucesivas apariciones. En ésta el director de la revista comunica detalladamente a Donoso la presencia que tendrá su obra en los siguientes números de la revista:

Acabo de recibir el prólogo para El obsceno pájaro de la noche, que me gusta mucho. Lo voy a publicar en el número siguiente al que sale una larga nota mía (ocho página de la revista) sobre el mundo de José Donoso. En esa nota trato de situar con la mayor profundidad posible tus dos novelas y también tus cuentos, y espero que sirva de buen

\footnotetext{
${ }^{10}$ Los avances de diferentes obras literarias como parte de una sección en Mundo Nuevo se inician con el segundo número, y se inauguran con un fragmento de Cien años de soledad. El primer avance del Obsceno pájaro, sin embargo, fue publicado en la revista Diálogos, en 1965. Para otro avance del Obsceno pájaro, fuera de Mundo Nuevo, ver Marcha 1217 (1964): s. pag. Print.
} ISSN 0034-9631 (Impreso) ISSN 2154-4794 (Electrónico) correctivo a las tonterias que se dicen por ahí, sobre todo en Chile. En estos momentos la operación Donoso consiste en los siguientes movimientos estratégicos:

Número de mayo: Artículo de Cristián Huneeus en que sintetiza el debate sobre la novela chilena aparecida en 'Ercilla';

Número de junio: con la consagración (o coronación) a cargo de ERM [Emir Rodríguez Monegal]. En el mismo número nota breve en 'Sextante', de Cristián Huneeus, con resumen de algunas opiniones chilenas sobre Este Domingo, y palos a los compatriotas;

Número de julio: publicación del prólogo de la próxima novela de Donoso. En el ínterin (sic) pienso extraerles a Carlos [Fuentes] y a [Severo] Sarduy los artículos que me han prometido. (13/4/67 José Donoso Papers UI)

Efectivamente, cada uno de los "movimientos estratégicos" recientemente enumerados se lleva a cabo. Primero, en la sección "Revistas" aparece el artículo de Cristián Huneeus, titulado “¿Existe la novela chilena?”. En éste, Huneeus sintetiza el debate generado por la respuesta de un número de críticos ante la interrogante "¿Qué sucede con la novela chilena?" y “¿Crisis de la novela chilena?" abiertas en la revista Ercilla y El siglo, respectivamente. Luego, da espacio para que algunos escritores den su opinión al respecto. Entre ellos, José Donoso (88-89), quien sostiene que la crisis y el estancamiento en cuestión no se aplican a la literatura de los nuevos escritores (generación en la que él se incluye abiertamente), sino a la anterior: aquella que si bien "tuvo sus buenos momentos ... indudablemente no hizo nada comparable a Cortázar, a Sábato, a Borges, a Onetti, ni a Rulfo" (88-89). Si bien el artículo da voz tanto a quienes argumentan a favor de una crisis en la literatura chilena y quienes por el contrario sostienen que se trata de un nuevo momento; el artículo de Huneeus claramente favorece la segunda postura.

En el mismo número, también aparece una extensa nota de Rodríguez Monega titulada "El mundo de José Donoso" (77-85), en la que primero presenta detalladamente al escritor y su obra. Luego analiza en profundidad las tres novelas hasta entonces publicadas -Coronación, Este Domingo y El lugar sin límites-proponiendo como tema constante en su obra "la violencia soterrada/escondida que repentinamente, y bajo diferentes formas, se libera" (81). Como es de esperar, el artículo se cierra retratando favorablemente a Donoso como un escritor "cuya plena madurez se inicia realmente ahora" (85). Por último, tal como Rodríguez Monegal señala en la carta que nos ocupa, Huneeus defiende Este domingo de la crítica chilena, en la sección "Sextante", titulada "Donoso en Chile" (94-95). Sus palabras desacreditan al sector de la crítica que no ha valorado la última novela de Donoso:
Revista Iberoamericana, Vol. LXXIX, Núms. 244-245, Julio-Diciembre 2013, 1049-1068 ISSN 0034-9631 (Impreso) 
La reciente publicación de Este Domingo, de José Donoso (Zig-Zag, 1966), tendrá que esperar su acogida en el exterior para ser valorada, en sus logros y limitaciones, con seriedad e inteligencia. La crítica chilena la ha despachado en crónicas sumarias, crudas y superficiales, que recorren el arco comprendido entre la adulación infundida por la creación mitológica del 'éxito en el extranjero' y la frustración de las expectativas, muy explícitas, con que el comentarista chileno aborda la lectura del Donoso. (94)

Quiero destacar en primer lugar que este compromiso con Donoso implica por parte de Rodríguez Monegal, un conocimiento tanto de la obra del escritor como de sus planes, información que obtiene mediante la correspondencia constante con el escritor. En segundo lugar, que su conocimiento no se limita a la información que el escritor provee en sus cartas, sino al constante envío de borradores que el crítico lee y comenta detenidamente.

En las cartas que Rodríguez Monegal recibe de Donoso, contenidas en los Emir Rodríguez Monegal Papers de la Universidad de Princeton, se puede acceder a la reacción del escritor ante el artículo en el cual el crítico ha hablado de su obra: "El tuyo es el primer artículo que siento que me toma entero, como escritor, como creador, no como historiador de costumbres" (7/7/67 Emir Rodríguez Monegal Papers, PU) Para comprender el entusiasmo de Donoso por ese no ser tomado "como historiador de costumbres", resulta necesario recurrir a la valoración que un sector de la crítica hace de la obra de Donoso, por su capacidad para retratar la realidad, y sobre todo a partir de Coronación, por su habilidad para recrear la decadencia de la burguesía criolla.

El origen de esta limitación se puede adjudicar, al menos parcialmente, a la inclusión de Donoso en la Generación del 50, categoría que reúne a un grupo de escritores jóvenes a partir de la antología elaborada por Enrique Lafourcade en 1954. Si bien esta colocación dentro de una nueva generación de escritores le asegura a Donoso un lugar dentro de la narrativa nacional, cuando aún era un escritor incipiente, tuvo el inconveniente de reducir el valor de la obra de Donoso a su capacidad para retratar la decadencia de la clase alta de la sociedad chilena del siglo xx. A partir de las palabras agradecidas de Donoso, Rodríguez Monegal responde pocos días después: "No sé si tendré éxito en lograr que tus compatriotas te elogien más pero estoy seguro que se van a sentir un poco picados en el amor propio por haber leído tus novelas en forma tan estúpida. Sarduy está terminando ya la nota sobre El lugar sin límites probablemente saldrá en la revista a la rentrée" (10/7/67 José Donoso Papers PU).

En el número siguiente (13) aparece el segundo avance enviado por Donoso para ser publicado y corresponde al Obsceno pájaro. El estudio que he hecho de los borradores mecanografiados pertenecientes al proceso de escritura de El obsceno pájaro de la noche, contenidos en los José Donoso Papers de la Universidad de Iowa, me permite afirmar que dicho fragmento pertenece a un conjunto de borradores mecanografiados que tuvo por título "Confabulación de vagabundos", y que en la obra publicada se incorpora parcialmente en la escena final de la novela. En una carta que Donoso recibe de Rodríguez Monegal en mayo del 67, se puede inferir que Donoso se ha arrepentido de enviar el fragmento escogido para su publicación: “Tu Carta de abril 22 llegó demasiado tarde y el número en que va El pájaro ya está compuesto y en la imprenta. Pero no te preocupes porque ese trozo, quede o no quede en la novela, es valioso por sí mismo y ayuda a abrir el apetito para el libro entero" (10/5/67 José Donoso Papers UI).

La respuesta de Rodríguez Monegal evidencia, en primer lugar, la finalidad con la que se crea la sección "libros": dar a conocer a sus lectores lo que los escritores están produciendo y prontos a publicar, para generar expectativas y sobre todo, interés en los lectores. Por otra parte, que para la revista, la publicación de un avance de una novela que resulta exitosa, constituyó un elemento de orgullo que su director resalta como un mérito. Por sólo citar un ejemplo, en el número 11 de 1967, se puede ver cómo el editor junto con celebrar que Carlos Fuentes recibiera el premio Biblioteca Breve en 1967 por Cambio de piel, destaca que "Los lectores de Mundo Nuevo ya conocen la novela que fue largamente discutida por su autor en una entrevista publicada en el núm. 1 (julio de 1966)" y que algunos de sus "capítulos se adelantaron en el núm. 4" (92).

Finalmente, en la sección "sextante" del número 15, último volumen en el que se materializa la "operación Donoso", aparece un breve apartado titulado "Donoso fija sus límites" (70), el que reproduce fragmentos de una entrevista por Carlos Alberto Cornejo, publicada cuatro meses antes en la revista Ercilla. El texto sin duda constituye un ejemplo del interés de Mundo Nuevo por traspasar "las barreras nacionales" para difundir la literatura latinoamericana, como se declara en el ideario de la revista que acompaña el primer número, ya que Ercilla sólo circulaba dentro de Chile. ${ }^{11}$

Por último, la correspondencia deja de manifiesto que el apoyo que Rodríguez Monegal brinda a Donoso, no se limita al período en el que dirige Mundo Nuevo. En el número 72 de la revista Review (1973), se dedica la sección "Focus" a la obra de Donoso. Si bien el editor de esta revista es Ronald Christ, este número cuenta con la participación de Rodríguez Monegal como “Contributing Editor". La sección se inaugura con una cronología de la vida y obra de Donoso, por el propio Donoso (12-19). La siguen cuatro artículos de Zunilda Gertel, Francisco Rivera, John Hasset, Severo Sarduy; y se cierra con la publicación de la reconocida entrevista de Rodríguez Monegal a Donoso sobre el Obsceno pájaro, "The Novel as a Happening: an Interview with José Donoso". En el caso de los artículos, la intervención de Rodríguez Monegal en este número se hace evidente en el texto de Sarduy, "Writing/ Transvestism" ya que éste fue previamente publicado en español, en Mundo Nuevo.

1 Por todo lo anterior, llama mi atención la ausencia de Donoso en la enumeración de los escritores que juicio de Ernesto Sierra tuvieron un lugar privilegiado en las páginas de Mundo Nuevo (12). Ver "Mundo Nuevo y la máscara de la cultura"

Revista Iberoamericana, Vol. LXXIX, Núms. 244-245, Julio-Diciembre 2013, 1049-1068
ISSN 0034-9631 (Impreso)

$\frac{\text { Vol. LXXIX, Núms. 244-245, Julio-Diciembre 2013, 1049-1068 }}{\text { ISSN 2154-4794 (Electrónico) }}$
Revista Iberoamericana, Vol. LXXIX, Núms. 244-245, Julio-Diciembre 2013, 1049-1068 ISSN 0034-9631 (Impreso) ISSN 2154-4794 (Electrónico) 
Las cartas interpretadas retratan a Rodríguez Monegal como un crítico que, plenamente consciente de su estatus dentro del circuito de escritores e intelectuales latinoamericanos, se encarga de empujar la difusión de la obra de Donoso en tres modalidades. A través de otros escritores o especialistas, mediante su labor docente y trabajo crítico, lo que se traduce principalmente en la inclusión de la obra del prosista en los cursos que dicta, los textos que escribe sobre narrativa latinoamericana, sugiriéndolo como invitado a las universidades donde trabaja, e incorporando la obra de Donoso a un canon que a lo largo de los años el crítico paulatinamente construye y refuerza.

\section{José Donoso y CARLos Fuentes: LA FRATERNIDAD DEL OFICIO DE LA ESCRITURA}

La correspondencia que Donoso recibe de Fuentes está marcada desde el principio por el oficio de la escritura. Se inaugura con una carta escrita en marzo de 1962, desde México D.F. (José Donoso Papers UI), en la que comenta con entusiasmo haber leído Coronación. Esto es a cinco años de la publicación de esta novela y después de que ambos escritores se reconocieran en el Congreso de intelectuales realizado ese mismo año en la ciudad de Concepción.

La mayoría de las cartas retratan una relación que en términos de dinámica no se diferencia mayormente de la que Donoso mantiene, a través de la correspondencia, con Rodríguez Monegal. De manera tal que es una interacción marcada por el apoyo continuo por parte de un escritor plenamente posesionado del lugar que ha adquirido dentro de la esfera de la producción literaria y la de un escritor cuya obra es todavía incipiente en 1962.

Para abrirle el paso a Donoso desde su posición privilegiada, Fuentes lee y comenta su obra en el espacio privado de la carta, y dentro de la esfera pública escribe reseñas y críticas que la favorecen. También la defiende, utilizando como tarima, por ejemplo, Mundo Nuevo u otras revistas, así como las entrevistas en las que participa. En una carta de abril de 1967, por ejemplo, se preocupa de reproducir una extensa cita de lo que dijo a su favor, durante una entrevista. En otra de 1971 le dice que espera saber su opinión sobre el Obsceno pájaro a través del artículo que ha escrito para Plural, "la nueva revista literaria dirigida por Paz y publicada por el diario 'Excélsior', cuyo primer número saldrá en septiembre [...] Espero que te guste" (27/7/71 José Donoso Papers PU).

Un ejemplo de esa lectura crítica en el ámbito privado se encuentra en la carta que inaugura la correspondencia contenida en los José Donoso Papers de la Universidad de Iowa. En ésta, Fuentes alaba y comenta con entusiasmo Coronación: "es, sin duda, una de las novelas más perfectas e importantes que se han escrito últimamente en español [...] me parece que en pocas novelas latinoamericanas he encontrado una exactitud mayor entre el material empleado y el lenguaje que lo expresa" (9/3/62 José Donoso Papers UI). Las palabras de Fuentes, sin embargo, no se limitan a ensalzar la novela: "Me molesta el largo diálogo explicativo entre Andrés y Carlos [personajes de

Revista Iberoamericana, Vol. LXXIX, Núms. 244-245, Julio-Diciembre 2013, 1049-1068 ISSN 0034-9631 (Impreso) ISSN 2154-4794 (Electrónico)
Coronación], que para mí es el único lunar de la obra; creo que no hacía falta" (9/3/62 José Donoso Papers UI). A partir de las palabras de Fuentes se materializa, por una parte, el interés que el escritor tiene en la obra de Donoso, así como una muestra de una lectura atenta que, como consecuencia, otorga credibilidad al elogio con el que inicia su comentario sobre la novela. Además, da cuenta de una relación entre dos escritores que sin duda se encuentran en puntos diferentes dentro de su carrera. Fuentes ha publicado lo suficiente como para emitir una opinión sobre lo que es valioso y lo que por el contrario es indeseable en términos de escritura. Donoso, por su parte, se encuentra en la posición del escritor incipiente, pero prometedor, al que la crítica de un prosista más experimentado y exitoso resulta valiosa en términos de autoestima y oficio.

Dentro del apoyo que Fuentes da a Donoso también cabe resaltar las medidas que, por iniciativa propia, toma para que ésta circule y sea traducida. En efecto, en la misma carta donde ha comentado Coronación, Fuentes expresa interés en que sea traducida: "Me parece GROTESCO que tu novela no esté traducida al inglés y al francés. Por favor envíame dos o tres ejemplares para que trabaje esto con mi agente en Nueva York y con la gente de Gallimard en París. Tengo la seguridad de que mi gestión no será en vano" (9/3/62 José Donoso Papers UI). Cabe señalar que a diferencia del fragmento que -a juzgar por el uso de las comillas-Donoso cita literalmente en Historia personal, donde es Donoso quien debe enviar su novela al agente literario de Fuentes en Nueva York; en el recientemente citado, es Fuentes quien se ofrece personalmente para llevar a cabo la gestión, ampliando además las posibilidades de una traducción al francés. A partir de la carta que Donoso recibe tres meses después, se desprende que el escritor respondió a la demanda, y que Fuentes por su parte, cumplió con su palabra: "[...] mi agente en Nueva York ya está manejando 'Coronación' y, aunque apenas está haciendo los 'rounds' de lectura entre críticos bilingües parece que se apunta la posibilidad de que Simon \& Schuster se interese por la obra" (17/6/62 José Donoso Papers UI). En relación a la entrada de Coronación al mercado editorial francés, confiesa no haber iniciado el trámite: "Respecto a Francia, voy a tomarme un poco más de tiempo para asegurar la edición con Gallimard". La razón del retraso, no obstante, sugiere que se debe a la peculiaridad de los editores y no a un descuido: “[...] los editores franceses sólo funcionan a base de influencias personales, de manera que voy a esperar la visita de Juan Goytisolo a México en septiembre para encargarle personalmente la gestión" (17/6/62 José Donoso Papers UI). La resolución de Fuentes devela otro rasgo significativo de la forma cómo apoya a Donoso, sobre todo en una primera etapa: utiliza su conocimiento sobre el funcionamiento del medio editorial y de los traductores, así como sus contactos, para empujar la obra del escritor chileno, sobre todo en la etapa en que ésta aún no ha salido de Chile. ${ }^{12}$

A juzgar por Historia personal, no fue Donoso quien respondió a la solicitud de Fuentes de enviar ejemplares de Coronación para comenzar las gestiones a favor de su traducción, sino su esposa Pilar: "A

Revista Iberoamericana, Vol. LXXIX, Núms. 244-245, Julio-Diciembre 2013, 1049-1068 ISSN 0034-9631 (Impreso) ISSN 2154-4794 (Electrónico) 
La carta que Donoso recibe cuatro meses después retrata la lentitud del proceso así como la competencia entre las editoriales interesadas en publicar nuevos escritores latinoamericanos: "Me escribe Brandt, mi agente de Nueva York; aunque Simon \& Schuster todavía no decide. 'Coronación' también está, ahora, en manos de Knopf. Estas cosas se toman su tiempo, de manera que paciencia. Lo importante es que el libro esté en manos de dos de las editoriales más importantes de los Estados Unidos" (2/10/62 José Donoso Papers UI)

La correspondencia, por otra parte, también devela una persistencia del plan original y a la vez grafica hasta qué punto Fuentes estaba inmerso e informado de lo que ocurría con las editoriales importantes, incluso cuando no estaba viviendo en París: "Cuando venga Goytisolo a México en noviembre lo alojaré y lo sentaré a leerte para que a su regreso a París lleve el libro ofrecido para Gallimard, que por fortuna ha suprimido a la folklórica colección ' $L a$ Croix du Sud' y ahora publicará a los latinoamericanos en 'Du Monde Entier'” (2/10/62 José Donoso Papers UI). ${ }^{13}$

Gracias a una carta de Fuentes a Donoso de noviembre del mismo año, podemos acceder al revés de la traducción al inglés de Coronación, ya que en ésta se materializa el resultado de las estrategias comentadas anteriormente. Resalta, en primer lugar, una interesante cadena en la que, si bien Donoso es el destinatario, Fuentes y su agente son los intermediarios. La carta se abre con un breve saludo al que sigue la cita del recado que Alfred Knopf envía, por medio de Brandt y Fuentes, a Donoso. Este es, el quere hacerse cargo de la traducción de Coronación y de publicarla. Además de comentar los términos del contrato, Fuentes aconseja a Donoso -al parecer por segunda vez- que contrate a Brandt como agente literario: "Espero que le hayas escrito a Brandt [...] y que le hayas solicitado sus servicios de agente, $[\ldots]$ es realmente de primera, cuida de tus intereses como si fueras su Julieta, pelea por ti, obliga a los editores a hacer publicidad, 'pica' a los críticos, etc." (12/11/62 José Donoso Papers UI).

La carta con fecha 19 de agosto de 1962 también resulta crucial para aprehender esa "generosidad" de Fuentes que Donoso apunta en su Historia personal. En ésta Fuentes le explica la relación entre Díez Canedo y Barral: "El mes entrante inicia sus publicaciones

pesar de que mi mujer me urgía, no hice lo que Fuentes me pidió: manda ejemplares a Carl D. Brandt, en Pesar Pero sin que yo lo supiera, mi mujer, que enton a del Brasil, hizo por su cuenta y escondida de mí los paquetes con los gruesos volúmenes amarillos, gastándose el presupuesto de una semana de calefacción en el franqueo aéreo, expidió no sé cuántos ejemplares" (64).

Mediante la utilización del adjetivo "folclórico" Fuentes descalifica la colección creada por Roger Calloi al regresar a Francia después de su estadía en Argentina. La colección no sólo incluye a escritores de América del Sur, sino cubanos (Alejo Carpentier, Guillermo Cabrera Infante y Lydia Cabrera), mexicano (Juan Rulfo, Martín Luis Guzmán Franco, Juan José Arreola, Fernando Benítez y Rosario Castellanos), a costarricense Marcos Ramírez y al guatemalteco Mario Monteforte Toledo.

Revista Iberoamericana, Vol. LXXIX, Núms. 244-245, Julio-Diciembre 2013, 1049-1068 ISSN 0034-9631 (Impreso) ISSN 2154-4794 (Electrónico)
Joaquín Díez Canedo, exgerente de producción del Fondo de Cultura Económica. Creo que te contaba en Chile que Joaquín está asociado directamente con Seix Barral de Barcelona e indirectamente con Einaudi (Roma), Grove Press (N.Y) y Gallimard, de manera que publicar con él, casi automáticamente, asegura la traducción" (19/8/62 José Donoso Papers UI). En otra carta escrita en diciembre del mismo año, Fuentes comparte con Donoso cuánto le ofreció Knopf por la publicación de La región más transparente, con la finalidad de que se dé cuenta de la calidad del contrato que le han ofrecido por Coronación: "Por favor cablegrafíale a Brandt diciendo que aceptas los términos del contrato (a mí sólo me adelantaron $\$ 500$ dls. por 'La región')". Además, se preocupa de comentarle el alcance de que Knopf haya aceptado su novela: "Creo que te has sacado la lotería apareciendo con Knopf, que es quizás la GRAN casa editorial gringa en estos momentos, y sin duda la que asegura un mayor caudal de repercusiones críticas en la prensa de los EE.UU., y el mayor interés de parte de las editoriales europeas: de Knopf vas a brincar al sueco, al polaco y el (sic) lapón" (12/11/62 José Donoso Papers UI).

Limitar la discusión de la relación de ambos escritores al período en que Donoso es asistido por Fuentes sería un error. Resulta indispensable apuntar que dentro de su desarrollo es posible observar dos etapas. Una primera, en la que se esboza una relación entre un escritor con experiencia y plenamente posesionado del lugar que ha llegado a tener dentro de la esfera de la producción literaria y un escritor incipiente que genera interés en el primero, propiciando su apoyo. Una segunda, en la que Donoso ya no necesita ser protegido por Fuentes, sino Fuentes beneficiado por su ayuda. Un buen ejemplo de ello se encuentra en una carta enviada desde México, en julio de 1971, en la que Fuentes pide autorización a Donoso para utilizar el prólogo que éste escribió para su novela: "quiero pedirte un gran favor: sé, aunque no lo conozco, que has escrito el prólogo para el 'Artemio Cruz' de Salvat; quisiera pedirte tu autorización para incluirlo como prólogo a la misma novela en Aguilar; vas en buena compañía, pues los demás prólogos a obras individuales son de Paz, Lezama Lima, Sarduy, Goytisolo (Juan)" (27/7/1971 José Donoso Papers PU). Una carta posterior, de septiembre del mismo año, permite inferir que Donoso no ha respondido a su petición: "si no me mandas tu el prólogo de ARTEMIO CRUZ para incluirlo chez Aguilar, nadie lo hará. Please, pues la opera omnia está en prensa ya" (4/10/71 José Donoso Papers PU). Como se puede inferir, Fuentes en vez de encargar el prólogo de su novela a otro escritor opta por insistir a Donoso, hecho que a mi parecer habla del lugar que Donoso ya ocupa en los 70 .

Conclusiones

La incorporación de una interpretación crítica de la correspondencia inédita de José Donoso, permite cuestionar el fenómeno de la consagración de este escritor en términos cronológicos. Si nos limitamos al recuento de Donoso en Historia personal, las características formales y el éxito de El obsceno pájaro de la noche(1970), su accidentado

Revista Iberoamericana, Vol. LXXIX, Núms. 244-245, Julio-Diciembre 2013, 1049-1068 ISSN 0034-9631 (Impreso) ISSN 2154-4794 (Electrónico) 
reconocimiento por parte de Seix Barral en 1969, así como la posición fronteriza en la que queda en la revisión del Boom que ofrece Rama en 1979; pareciera que Donoso sólo logró unirse en el ocaso del Boom al "club más exclusivista que haya conocido la historia cultural de América Latina" (Rama 26). El estudio de su intercambio epistolar, por el contrario, revela a un José Donoso que no se suma al Boom a fines de la década del sesenta-principios de los setenta, sino que es tempranamente incorporado por dos figuras cruciales: Emir Rodríguez Monegal y Carlos Fuentes.

Por otra parte, la relación entre Emir Rodríguez Monegal y Donoso adquiere una nueva dimensión que ni el testimonio del escritor en su Historia personal del boom ni El boom de la novela Latinoamericana materializan. Esta es, la de un temprano y constante apadrinamiento por parte del crítico uruguayo, quien a mediados de la década de los sesenta aprovecha su posición de profesor visitante en una importante universidad norteamericana, y más tarde la de director de una revista literaria, para impulsar la obra y presencia de Donoso en un escenario internacional. Un segundo aspecto que el intercambio epistolar entre ambos deja en evidencia, es que Rodríguez Monegal se interesa tempranamente en la obra de Donoso. Esto es, al menos dos años antes de que el crítico estuviera a cargo de Mundo Nuevo y cuando la obra de Donoso se limitaba a un conjunto de cuentos y una novela breve. Así mismo, también se hace patente que el interés que Rodríguez Monegal tiene en Donoso no se limita a que el escritor envíe material inédito para que sea publicado en la sección de la revista dedicada a este fin sino en la experiencia lectora y capacidad crítica del escritor.

A partir del análisis de la correspondencia con Fuentes, atribuyo el interés del escritor mexicano en la obra de Donoso a la hermandad del oficio de la escritura. Como se ha podido ver en las cartas interpretadas, Fuentes se perfila como un escritor que a mediados de la década de los 60 ya goza de una posición en el medio editorial, que decide aprovechar su experiencia para aconsejar y compartir información relevante con un escritor entonces incipiente, propiciando la traducción de Coronación al inglés y más adelante, la publicación de El lugar sin límites en México. Su asistencia, sin embargo, no se limita a ayudarlo a superar el mercado editorial chileno, sino que además se materializa en una crítica favorable de su obra, en su inclusión en las diversas actividades en las que participaban esa nueva generación de escritores que reclamaban un nuevo espacio para la literatura latinoamericana dentro y fuera del continente.

El porqué del interés que Rodríguez Monegal tiene en Donoso a principios de la década de los sesenta, no es un hecho que la interpretación de la correspondencia alumbre directamente. Tomando en consideración los conflictos que Rodríguez Monegal enfrentó antes y durante la publicación de Mundo Nuevo, propongo dos hipótesis. La primera es que reconoció en Donoso a un escritor prometedor, cuya obra merecía ser valorada y reconocida. La segunda, en cambio, tiene relación con el hecho de que Donoso no se

Revista Iberoamericana, Vol. LXXIX, Núms. 244-245, Julio-Diciembre 2013, 1049-1068 ISSN 0034-9631 (Impreso) encontraba "manchado" políticamente ${ }^{14}$ y que por tanto estaría dispuesto -al menos en teoría-a colaborar con la revista mediante avances de sus novelas en composición; con reseñas y artículos críticos de otros escritores latinoamericanos contemporáneos. De este modo, la calidad de la obra de Donoso, su experiencia lectora y crítica, le permitiría recibir material contundente y de calidad para ser publicado en diferentes números. ${ }^{15}$

Por último, la propuesta de Luz Rodríguez-Carranza en relación a la agenda que Rodríguez Monegal impone en Mundo Nuevo, permite sumar una tercera hipótesis en relación al porqué del interés que el crítico uruguayo tiene en la obra de Donoso. A su parecer en Mundo Nuevo lidera la concepción de la literatura como "creación total y gratuita" (910), como ficción que no reproduce "la realidad según los códigos a los que estamos más acostumbrados" (914). Al alero de esta propuesta, el ofrecer artículos críticos que rescataran el valor que la crítica chilena no supo identificar en la obra de Donoso, calzaba a la perfección con la agenda que el director de la revista impuso en su publicación periódica, al permitirle luchar contra la concepción de la literatura como reproducción de la realidad y por ende de la renovación de la literatura latinoamericana.

Jacques Joset en "El imposible boom de José Donoso" propone la comprobación de la propuesta de Donoso en relación a la "importancia de Carlos Fuentes en tanto institución literaria" como una tarea pendiente:

Las relaciones personales entre el novelista mexicano y el chileno bien pueden influir en la visión de éste de tal forma que, quizá, sea exagerada la importancia de Carlos Fuentes en tanto institución literaria definida como 'uno de los factores precipitantes del boom'. [...] Las correcciones eventuales o la comprobación de la tesis donosiana pertenecen a una historia 'institucional' del boom todavía por hacer. (98)

Las cartas incluidas en este estudio no solo proveen ejemplos concretos que confirman y amplían el testimonio de Donoso en su Historia respecto de la significativa intervención que Fuentes tuvo en su carrera, sino que también subrayan la relevancia de estudiar la correspondencia personal de este escritor como una de las fuentes de investigación propicias para determinar los alcances de su agencia en la internacionalización de la literatura latinoamericana. ${ }^{16}$

${ }^{14}$ La falta de compromiso político es un hecho que Donoso se encargó de subrayar en su Historia personal, donde explícitamente contrapone su "propia congénita tibieza política" a la total adición de Fuentes y Vargas Llosa (60).

15 En "Breve ficha técnica de 'Mundo Nuevo', Mudrovic señala que en promedio se publicaron diez

números anuales durante los cinco años que se publicó la revista (44).
${ }^{16}$ Para una reflexión crítica sobre las posibilidades interpretativas que ofrece el estudio de la correspondencia 16 Para una reflexion critica sobre las posibilidades interpretativas que ofrece el estudio de la correspondencia
contenida en la Universidad de Princeton, ver "Corresponding Archives: Letters from the Latin American Literary Front" de Lucille Kerr.

Revista Iberoamericana, Vol. LXXIX, Núms. 244-245, Julio-Diciembre 2013, 1049-1068 ISSN 0034-9631 (Impreso) ISSN 2154-4794 (Electrónico) 
A la luz de las numerosas colecciones de archivo con los que contamos en el presente, el temor que Donoso expresó en "Diez años después" respecto de la carencia de materiales que permiten al investigador ahondar en la dimensión privada del boom, es hoy más bien un reto:

Todos, menos Carlos Fuentes, fueron siempre pésimos corresponsales y no se tiene noticia de diarios ni papeles secretos: así, del boom y sus intimidades no quedarán testimonios escritos porque se viaja demasiado y se habla demasiado por teléfono. No sucederá como con Bloomsbury, cuya vida total se puede reconstruir casi paso a paso, en relaciones sociales, amistosas y eróticas, por medio de diarios y correspondencias y recuerdos escritos. Las veleidades del corazón, en el boom, quedarán quizá siempre en secreto. $(217-218)$

El estudio de la correspondencia entre los escritores, editores, traductores y críticos relacionados con la internacionalización de la literatura latinoamericana durante la década del sesenta, permite acercarnos a la maquinaria detrás de uno de los períodos más fascinantes y controversiales en la historia cultural de América Latina, al constituir una valiosa fuente de información que permite -entre otras-ampliar, afirmar o refutar las propuestas obtenidas mediante otras fuentes relacionadas con el circuito literario de la nueva novela del Boom y la crítica elaborada por los propios protagonistas de este período. El análisis e interpretación de la correspondencia de José Donoso aquí realizada es tan solo un ejemplo del aporte que la inclusión de este material ofrece particularmente para el estudio de la red entre sus protagonistas.

\section{OBRAS CITADAS}

Christ, Donald, Leonora Saurel, Rosario Santos, Emir Rodríguez Monegal, eds. Review 73 (1973): 12-39.

Cortínez, Verónica. "La parroquia y el universo: Historia personal del boom de José Donoso". Revista chilena de literatura 48 (1996): 13-22.

Donoso, José. Historia personal del boom. Barcelona: Anagrama, 1972. Historia personal del boom. Santiago de Chile: Alfaguara, 1998.

"El obsceno pájaro de la noche". Marcha 1217 (1964): s.pag.

"El obsceno pájaro de la noche". Diálogos 1/3 (1965): s. pag.

"El obsceno pájaro de la noche". Mundo Nuevo 13 (1967): 14-22.

Huneeus, Cristián. "Donoso en Chile". Mundo Nuevo 12 (1967): 94-95.

“¿Existe la novela chilena?” Mundo Nuevo 12 (1967): 88-89.

"Donoso fija sus límites". Mundo Nuevo 15 (1967): 70.

Gerdes, Dick. "The Boom in Spanish American Literature. A Personal History by José Donoso; Gregory Kolovakos.” The Modern Language Journal 6/8 (1977): 444-445.

Revista Iberoamericana, Vol. LXXIX, Núms. 244-245, Julio-Diciembre 2013, 1049-1068 ISSN 0034-9631 (Impreso) ISSN 2154-4794 (Electrónico)
Hasset, John J. "Historia personal del 'boom' Personal History of the 'Boom' by José Donoso." Latin American Literary Review 2/3 (1973): 108-111.

Josset, Jacques. "El imposible Boom de José Donoso". Revista Iberoamericana 48 (1982): 91-101.

"La estrategia autobiográfica de José Donoso en Historia personal del 'boom" ", Crítica semiológica detextos literarios hispánicos: Actas del Congreso Internacional sobre semiótica e hispanismo, Madrid, 20-25 de junio 1983. Miguel Ángel Garrido Gallardo, ed. Madrid: Consejo Superior de Investigaciones Científicas II, 1986. 641-648.

Kerr, Lucille. "Corresponding Archives: Letters from the Latin American Literary Front." Symposium (2007): 75-96.

McMurray, George. "Historia personal del 'boom' by José Donoso.” Books Abroad 47/4 (1973): 736-737.

Mudrovcic, María Eugenia. Mundo Nuevo: cultura y Guerra Fría en la década del 60. Rosario: Beatriz Viterbo Editora, 1997.

Rama, Ángel. “El ‘Boom'en perspectiva”. Escritura: Revista de teoría y crítica literarias 4/7 (1979): 3-45.

Rodríguez-Carranza, Luz. "Emir Rodríguez Monegal o la construcción de un Mundo (Nuevo) posible". Revista Iberoamericana LVIII/160-161 (1992): 903-917.

Rodríguez Monegal, Emir, “El mundo de José Donoso”. Mundo Nuevo 12 (1967): 77-85. "José Donoso: la novela como 'happening". Revista Iberoamericana 37/74 (1971): 518-36

El 'boom'de la novela latinoamericana. Caracas: Editorial Tiempo Nuevo, 1972. Sarduy, Severo. "Escritura y travestismo". Mundo Nuevo 20 (1968): 72-74.

Sierra, Ernesto. "Mundo Nuevo y las máscaras de la cultura". Hipertexto 3 (2006): 3-13.

\section{CORRESPONDENCIA}

Donoso, José. Carta a Emir Rodríguez Monegal. 7 julio 1967. TS. José Donoso Papers Department of Rare Books and Special Collections, Princeton University Library. Carta a Emir Rodríguez Monegal. 11 octubre 1972. TS. Emir Rodrígez Monega

Papers. Department of Rare Books and Special Collections, Princeton University Library.

Fuentes, Carlos. Carta a José Donoso. 9 marzo 1962. TS. José Donoso Papers. Deparment of Special Collections, University of Iowa.

Carta a José Donoso. 17 junio 1962. TS. José Donoso Papers. Department of Special Collections, University of Iowa.

Carta a José Donoso. 19 agosto 1962. TS. José Donoso Papers. Department of Special Collections, University of Iowa.

Revista Iberoamericana, Vol. LXXIX, Núms. 244-245, Julio-Diciembre 2013, 1049-1068 ISSN 0034-9631 (Impreso) ISSN 2154-4794 (Electrónico) 
Carta a José Donoso. 2 octubre 1962. TS. José Donoso Papers. Department of Special Collections, University of Iowa.

Carta a José Donoso. 12 noviembre 1962. TS. José Donoso Papers. Department of Special Collections, University of Iowa.

Carta a José Donoso. 27 julio 1971. TS. José Donoso Papers. Department of Rare Books and Special Collections, Princeton University Library.

Carta a José Donoso. 4 octubre 1971. TS. José Donoso Papers. Department of Rare Books and Special Collections, Princeton University Library.

Rodríguez Monegal, Emir. Carta a José Donoso. 18 marzo 1965. TS. José Donoso Papers. Department of Special Collections, University of Iowa.

Carta a José Donoso. 31 marzo 1965. TS. José Donoso Papers. Department of Special Collections, University of Iowa.

Carta a José Donoso. 7 noviembre 1965. TS. José Donoso Papers. Department of Special Collections, University of Iowa.

Carta a José Donoso. 18 enero 1966. TS. José Donoso Papers. Department of Special Collections, University of Iowa.

Carta a José Donoso. 6 julio 1966. TS. José Donoso Papers. Department of Special Collections, University of Iowa.

Carta a José Donoso. 13 abril 1967. TS. José Donoso Papers. Department of Special Collections, University of Iowa.

Carta a José Donoso. 10 mayo 1967. TS. José Donoso Papers. Department of Special Collections, University of Iowa.

Carta a José Donoso. 10 julio 1967. TS. José Donoso Papers. Department of Rare

Books and Special Collections, Princeton University Library.

Carta a José Donoso. 23 agosto 1968. TS. José Donoso Papers. Department of Rare Books and Special Collections, Princeton University Library.

Carta a José Donoso. 11 octubre 1968. TS. José Donoso Papers. Department of

Rare Books and Special Collections, Princeton University Library. 